\title{
On the Role of the AIS Practitioner
}

\author{
Emma Hart, Mark Read, Chris McEwan, Julie Greensmith, Uwe Aickelin
}

The AIS field, which seeks to understand immune system operation and exploit these principles in engineering contexts, has been critized for "reasoning by metaphor" [Stepney 05]: algorithms are based on naïve biological analogies, are poorly understood, and weekly capture their inspiring biological properties. Various frameworks have been proposed that enable the richness of emergent biological properties to be translated into useful engineered systems. The conceptual framework [Stepney 05] proposed starting with a study of the immunological system, modeling it, and leading to the development of engineering algorithms. [Andrews 08] notes that the framework lacks guidance on selecting biological inspiration for particular engineering domains, and that evaluating a particular domain's potential requires that it first be modeled. The immuno-engineering framework [Timmis 08], through better grounded in engineering by accounting for the physical properties of engineering systems (e.g. in terms of memory, processing power etc.), suffers the same problem.

The interdisciplinary approach to algorithmic development advocated by these frameworks is essential but raises practical issues: immunologists inform modeling efforts to realistically represent and aid biological understanding; engineers desire algorithms that can be theoretically analyzed, verified and validated to demonstrate desirable properties and applicability to the problem at hand - the biological inspiration is irrelevant. Furthermore, engineers and immunologists will rarely interact; they have differing goals.

We propose redefining the term 'AIS practitioner', as an individual who identifies those components and interactions captured in computational immunology models that are responsible for a particular property of interest (POI), and to distil from these a set of algorithms and principles that can be applied in an engineering domain.

The cross-disciplinary AIS practitioner possesses sufficient understanding to identify key modern-day engineering challenges, and appreciate how qualities exhibited by the immune system can help. They can comprehend computational immunology models, and derive therefrom the required algorithmic principles. Stripping away immunological nomenclature is vital; algorithmic components labeled "T cells" or "lymph nodes" are so abstract at this stage that they are meaningless and confusing.

We capture the AIS practitioner's role as follows:

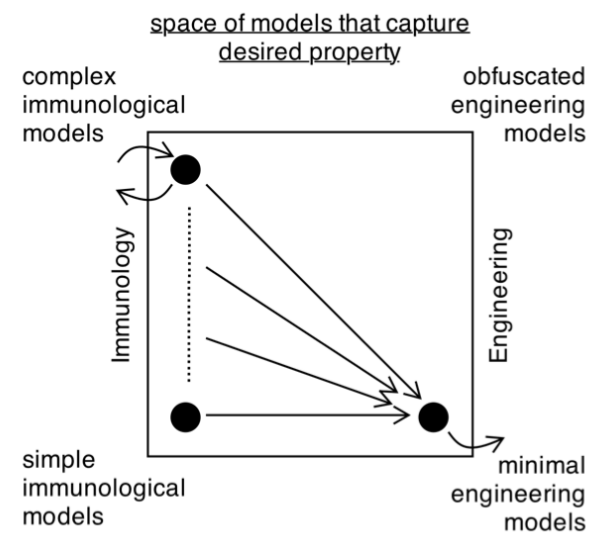

The box represents the space of abstract representations (models, simulations, algorithms, design principles) that capture a particular POI, a quality observed in the immune system and desired in an engineering solution, such as robustness, homeostasis, life-long learning, and adaptation, to name some examples [Hart 08]. Abstract representation complexity is represented vertically, whereas the domain of concern is depicted horizontally.

The bottom right corner represents the minimal rational representation (MRR), the minimal set of components/actors, interactions or principles responsible for manifestation of the POI, expressed free of immunological terminology. Its minimalism reflects the computational power and memory constraints of many engineering domains. The top right corner represents inefficient, sub-optimal and/or obfuscated abstract representations. Although they capture the POI, they contain superfluous interactions or components, and as such are not considered as suitable for adoption in engineering contexts. The top left 
contains detailed and complex models, representing, for example, large numbers of cells, pathways and spatial compartments. They may capture a great many immunological properties, in addition to the POI. The bottom left corner contains the minimal set of components and interactions responsible for the POI, and no other properties. Though minimal, these abstract representations exist in a form to benefit immunology, not in a form immediately applicable in engineering domains.

We consider the AIS practitioner's role to derive the MRR, given some abstract representation occupying the left of the box. The components and interactions present that are redundant or unnecessary with respect to the POI are removed or abstracted. Depending on the nature of the POI, and the immune-inspired engineering solution being derived, the MRR may be expressed as an algorithm, or as design principles for constructing systems.

\section{References}

P Andrews. An investigation of a methodology for the development of artificial immune systems: a casestudy in immune receptor degeneracy. PhD Thesis, the University of York, 2008.

S Stepney, RE Smith, J Timmis, AM Tyrrell, MJ Neal and ANW Hone. Conceptual frameworks for artificial immune systems. International Journal of Unconventional Computing 1(3):315-318, 2005.

J Timmis, E Hart, A Hone, M Neal, A Robins, S Stepney, A Tyrrell. Immuno-Engineering. 2nd IFIP International Conference on Biologically Inspired Collaborative Computing, 20th IFIP World Computer Congress, Milan, Italy, September 2008. IEEE Press Vol: 268/2008 pp. 3-17. 2008.

E Hart, J Timmis. Application areas of AIS: the past, present and future. Journal of Applied Soft Computing 8(1): 191-201, 2008 . 